\title{
Effectiveness of Daily Prednisolone to Slow Progression of Human T-Lymphotropic Virus Type 1-Associated Myelopathy/Tropical Spastic Paraparesis: A Multicenter Retrospective Cohort Study
}

\author{
Ariella L. G. Coler-Reilly ${ }^{1} \cdot$ Tomoo Sato $^{1} \cdot$ Toshio Matsuzaki $^{2} \cdot$ Masanori Nakagawa $^{3}$. \\ Masaaki Niino $^{4}$ - Masahiro Nagai ${ }^{5}$ - Tatsufumi Nakamura ${ }^{6}$ - Norihiro Takenouchi ${ }^{7}$. \\ Natsumi Araya $^{1} \cdot$ Naoko Yagishita $^{1} \cdot$ Eisuke Inoue $^{8} \cdot$ Yoshihisa Yamano $^{1}$
}

Published online: 23 May 2017

(C) The American Society for Experimental NeuroTherapeutics, Inc. 2017
Yoshihisa Yamano

yyamano@marianna-u.ac.jp

1 Department of Rare Diseases Research, Institute of Medical Science, St. Marianna University School of Medicine, Kanagawa, Japan

2 Department of Neurology and Geriatrics, Kagoshima University Graduate School of Medical and Dental Sciences, Kagoshima, Japan

3 Department of Neurology, North Medical Center, Kyoto Prefectural University of Medicine, Kyoto, Japan

4 Department of Clinical Research, Hokkaido Medical Center, Sapporo, Japan

5 Department of Neurology and Clinical Pharmacology, Ehime University Graduate School of Medicine, Ehime, Japan

6 Department of Social Work, Faculty of Human and Social Studies, Nagasaki International University, Nagasaki, Japan

7 Department of Microbiology, Kansai Medical University, Osaka, Japan

8 Medical Informatics, St. Marianna University School of Medicine, Kanagawa, Japan evaluate 57 patients treated with low-dose PSL (mean $4.8 \mathrm{mg}$ /day) versus 29 untreated patients. Roughly half of the evaluations spanned $<3$ years (Short-Term) and half $>$ 3 years (Long-Term), with a mean of 3.4 years. While the OMDS of most untreated patients remained unchanged in the Short-Term (87\%) and worsened in the Long-Term (79\%), most treated patients improved in the Short-Term (52\%) and remained unchanged or improved in the LongTerm (68\%). Overall, the mean change in OMDS per year was -0.13 in the Steroids group and +0.12 in the Untreated group $(p<0.01)$. This study addressed the effectiveness of PSL for HAM/TSP in 3 novel ways: 1) continuous low-dose administration; 2) comparison with an untreated group; and 3) Long-Term evaluation. These findings provide robust evidence supporting PSL maintenance therapy for HAM/TSP.

Keywords HTLV-1 $\cdot$ HAM/TSP $\cdot$ Myelopathy · Prednisolone $\cdot$ Corticosteroids $\cdot$ OMDS

\section{Introduction}

According to conservative estimates, there are 5 to 10 million individuals infected with human T-lymphotropic virus type 1 (HTLV-1) worldwide, including at least 1 million in Japan $[1,2]$. In addition to Japan, the virus is endemic to the Caribbean area and certain foci in South America, intertropical Africa, and the Middle East, especially north-eastern Iran. Indeed, Japan is considered the only highly developed nation severely afflicted, with most other disease foci in medically underserved tropical areas.

A small fraction of those infected develop the progressive neurodegenerative disease HTLV-1-associated myelopathy/ tropical spastic paraparesis (HAM/TSP) [3]. HAM/TSP is 
characterized by chronic spinal cord inflammation with accompanying myelopathic symptoms, mainly affecting the lower body. One of the hallmarks of HAM/TSP is progressively worsening motor disability, which is often assessed using the Osame Motor Disability Score (OMDS) [4], a scale ranging from 0 to 13 (Table 1). Other symptoms include back and leg pain, weakness and stiffness in the legs, numbness and tingling in the legs, urinary urgency and incontinence, constipation, and erectile dysfunction.

Prednisolone therapy for HAM/TSP dates all the way back to the definition of the disease in 1986 [5], and the stage had already been set for an anti-inflammatory approach long before. In the 1960s, prior to the revelation that HTLV-1 was the causative agent [5-7], when the disorder was still characterized only vaguely as TSP or Jamaican neuropathy, autopsies showed that the mysterious symptoms were due to an "inflammatory disease" primarily affecting the spinal cord $[8,9]$. Corticosteroids, synthetic versions of natural steroid hormones, had burst onto the scene in the 1940s and were becoming the most widely used and effective treatments for inflammatory conditions $[10,11]$. They act mainly by downregulating multiple inflammatory genes, such as those encoding cytokines and chemokines, that may have been activated in the chronic inflammatory process. Prednisone, and its active metabolite prednisolone (the form of the drug commonly administered in Japan), is probably the most widely used, accessible, and affordable corticosteroid for systemic administration; its high glucocorticoid activity makes it a particularly effective anti-inflammatory and immunosuppressive agent. By the 1980s, these and the intravenous (IV) form, methylprednisolone (as well as other immunosuppressants) were already being widely tested in patients with multiple sclerosis [12-14], an autoimmune disease of the central nervous system that can present with similar symptoms of

Table 1 Osame Motor Disability Score

\begin{tabular}{ll}
\hline OMDS & Description \\
\hline 0 & No walking or running abnormalities \\
1 & Normal gait but runs slowly \\
2 & Abnormal gait (stumbling, stiffness) \\
3 & Unable to run \\
4 & Needs handrail to climb stairs \\
5 & Needs a cane (unilateral support) to walk \\
6 & Needs bilateral support to walk \\
7 & Can walk 5-10 m with bilateral support \\
8 & Can walk 1-5 m with bilateral support \\
9 & Cannot walk, but able to crawl \\
10 & Cannot crawl, but able to move using arms \\
11 & Cannot move around, but able to turn over in bed \\
12 & Cannot turn over in bed \\
13 & Cannot even move toes \\
\hline
\end{tabular}

neurodegeneration. Accordingly, prednis(ol)one became the first treatment for HAM/TSP, a chronic neuroinflammatory condition with unknown etiology, and remains the most common treatment for the disease today $[3,15]$.

Despite the longstanding use of corticosteroid therapies for HAM/TSP, their effectiveness is controversial. There has never been a placebo-controlled trial, and the results from case series and other open-label studies are varied. The findings of such studies will now be briefly reviewed.

First, Osame et al. $[5,16]$ reported all patients $(n=4)$ exhibited "dramatic improvement" after high-dose (30-60 mg/day) oral prednisolone (PSL) and remained stable on a much lower maintenance dose when tapered slowly. That same year, 1987, Vernant et al. [17] reported in passing that PSL, taken by 2 patients at an unspecified dosage, showed "questionable effect," and this study was later cited as evidence for the ineffectiveness of PSL [18-20]. Comparable studies mentioning anecdotal accounts of PSL ineffectiveness abounded [21-24]. In a similarly contentious vein, patients on 5-day regimens of high-dose IV methylprednisolone (mPSL) were improved ( $n=$ $2)$ in 1 study [25] and unchanged $(n=3)$ or only transiently benefitted $(n=5)$ in another within the same year [26]. The latter study was reinforced when Araújo et al. [19] tried a 5day course of $1 \mathrm{~g}$ IV mPSL in 23 patients with similar results: improvement in pain that declined again within 60 days and no effect on Disability Status Scale (DSS) score. In 1990, Osame et al. [4] put forth the first substantial cohort $(n=65)$ of patients treated with PSL, starting at 60 to $80 \mathrm{mg}$ every other day for 2 months, tapering down to $5 \mathrm{mg}$ over 6 months, and then ceasing treatment 3 months later. They reported that only 6 patients failed to respond favorably, with many improving by $1(n=24)$ or even 2 ( $n=13)$ OMDS grades; while patients declined after discontinuing treatment, they retained some degree of benefit up to 6 months later. Soon after, Kira et al. [27] reported that patients on 40 to $60 \mathrm{mg}$ PSL over 1 to 4 months $(n=16)$ showed only minor, subjective benefits that disappeared upon long-term follow-up (roughly 2 years following cessation of treatment) and no change in DSS. By 1996, the Osame group had expanded their PSL cohort to 131 patients: they again reported that PSL was effective, reducing OMDS in $69.5 \%$ of patients, and also concluded that PSL was more effective than several other immunotherapies they had tested; however, they conceded that the changes were, indeed, transient [20]. After a decade of relative quiet, Croda et al. [28] reported in 2008 that repeated 3-day courses of $1 \mathrm{~g} \mathrm{IV} \mathrm{mPSL}$ produced changes in Incapacity Status Scale but neither OMDS nor DSS after roughly 2 years $(n=39)$.

Importantly, none of these studies employed a control group such that the progression of motor disability was compared between substantial cohorts of patients treated with PSL (or IV mPSL) and those receiving no immunotherapy. There were also no long-term studies evaluating the effects of PSL taken continuously over several years. 
In the present study, we remedy these substantial knowledge gaps, providing important information for treating this rare and devastating disease effectively. We hypothesized that steady low-dose oral PSL effectively slows the progression of HAM/TSP, and we tested this by measuring changes in OMDS with and without treatment over several years in a multicenter retrospective study.

\section{Methods}

\section{Study Design}

This was a multicenter retrospective cohort study using data collected by physicians monitoring patients with HAM/TSP in hospitals throughout Japan. The objective was to determine if daily low-dose oral PSL ameliorates the symptoms and slows the progression of HAM/TSP. The outcome measure was change in OMDS.

\section{Recruitment and Registration}

Requests to perform a collaborative study were sent by email to contacts at 13 institutions in Japan that were expected to be actively monitoring patients with HAM/TSP. Out of those institutions, 6 responded favorably to our request. Therefore, including our own St. Marianna University School of Medicine, 7 institutions participated in this study. At the participating institutions, the attending physicians filled out questionnaires for each of their patients with HAM/TSP who appeared to fit our inclusion criteria (see "Subjects and Data Curation" subsection) using information from their medical charts. They sent these completed forms to our office either electronically or by mail.

\section{Ethical Guidelines, Patient Privacy, and Informed Consent}

The study was approved by the St. Marianna University School of Medicine Bioethics Committee (Approval Number: 2254) as well as by the ethics committees of the 6 other participating institutions. In accordance with all ethical guidelines, the principle investigator at each institution made patients aware of this study using posters we provided and gave all patients the opportunity to refuse to participate. All data used in this study were collected during routine medical examinations, and no new interventions were performed on the patients for the purposes of the study. Patient information was anonymized at each institution before sending out the data by assigning patient identification numbers, and no individual can be identified from the information published in this study. Thus, it was not necessary to obtain individual informed consent, and appropriate precautions were taken to ensure the protection of patient privacy. Patient information was not used for anything other than the purposes outlined in this study.

\section{Data Collection}

Data were collected between 20 December 2012 and 31 March 2015. These data included: basic information (age, sex, date of birth, and institution at which the patient was monitored), the dates of each milestone (disease onset, first visit at the institution, start of treatment for treated subjects, and most recent visit at the institution), the OMDS recorded at each of those milestones, presence or absence of complications (especially those affecting motor ability), and treatment history (the dosage of PSL and/or interferon (IFN)- $\alpha$, which are the pharmaceutical drugs used to treat HAM/TSP-related inflammation in Japan).

\section{Patients and Data Curation}

Patients who met the inclusion criteria were registered as subjects in this study. These were patients who had been diagnosed with HAM/TSP according to the World Health Organization criteria [29] after cerebrospinal fluid testing and who had been monitored at one of the participating institutions for at least 1 year. Patients were excluded if the attending physician determined that sufficient information to answer the questionnaire could not be obtained.

We received data from 127 patients in total, 13 of whom were then excluded from the data analysis in this paper for the following reasons: insufficient OMDS records (7 patients); lack of motor disability, that is, an OMDS of 0 ( 2 patients); presence of other conditions that affect motor ability ( 1 patient with Parkinson's disease, 1 with hip osteoarthritis); low cerebrospinal fluid antibody titer (1 patient); and problems confirming the accuracy of the data obtained (1 patient).

The remaining 114 patients were divided into three groups based on their treatment status during the monitoring period: 57 patients who were continuously treated with PSL only, 29 who were untreated (neither with steroids nor interferon-alpha), and 28 other patients. This group of 28 patients was composed of 12 who discontinued PSL treatment; 2 who were treated with IFN- $\alpha$; and 14 who were treated with both PSL and IFN-alpha, including 5 who discontinued treatment. In this paper, the PSL group is compared with the untreated group, and the 28 other patients are not included in any analysis. Thus, 86 patients were analyzed as subjects in this study.

\section{Calculations, Variables, and Statistical Analysis}

Patients in the study were evaluated based on the following variables: dosage of PSL, period of evaluation, and OMDS. The average daily dose of PSL taken by each of the 57 patients treated during the monitoring period was calculated as a 
simple average of each recorded dose in the medical records without factoring in the number of days spent at each recorded dose. It was not possible to calculate a weighted average due to the limited data available. In cases where patients were treated every other day instead of every day, the dose was halved to calculate the daily dose.

The start of the period of evaluation was defined as the initial visit at the monitoring institution for those in the Untreated group and as the first day of treatment for those in the Steroids group. The end of the period of evaluation was the date of the final visit at the monitoring institution before the patient's data were transferred to us. The period of evaluation is listed in terms of years but was calculated to the day, such that, for example, the time from 1 January 2000 to 31 December 2000 would be recorded as 0.99 years rather than 0 years.

Patients were divided into groups based on period of evaluation; we decided to divide them into "Short-Term" ( $<3$ years) and "Long-Term" ( $>3$ years) groups because reports indicated that changes in OMDS typically appear over 3 years, meaning that a typical untreated patient evaluated over at least 3 years would be expected to exhibit an increase in OMDS [30].

The following potential confounders were identified and evaluated: sex, institution where the patient was monitored, age, and disease duration. Age at start was calculated to the day similarly to period of evaluation. Disease duration at start, that is, the time from onset to start, was calculated using the maximum precision possible for each patient: if only the year of onset was listed, disease duration was calculated roughly by subtracting the start year from the onset year $(n=78)$; if the date of onset was listed in a specific month, disease duration was also calculated to the month $(n=8)$.

Categorical variables (sex, institution, short- or long-term period of evaluation, direction of OMDS change) were compared using Fisher's exact test. Numeric variables (age, disease duration, period of evaluation, quantity and direction of OMDS change) were compared using the Wilcoxon rank sum test. Changes in OMDS by baseline score were compared using a stratified version of the Wilcoxon rank sum test, the van Elteren test. The strata for this analysis were baseline OMDS 3 to 4, 5, 6 to 7, and 8 or more. Statistical analyses were performed using R Version 3.3 and JMP®, Version 12 (SAS Institute Inc., Cary, NC, USA).

\section{Results}

\section{Average Daily Dose of Steroids for Treated Patients}

Out of the 86 patients analyzed in this study, 57 were continuously treated with PSL while under the care of the attending physician who supplied the data from their medical records. Using that data, we approximated an average daily dose of PSL for each patient. This daily dose ranged from 2.3 to
$11.7 \mathrm{mg} /$ day, and the median daily dose for all 57 patients was $4.8 \mathrm{mg}$ /day (Fig. 1A).

\section{Attributes of Patients in the Steroids and Untreated Groups}

In order to assess the effects of the steroid treatment, the progress of the aforementioned patients in the Steroids group ( $n=$ 57) was compared with that of the patients in an Untreated group ( $n=29$ ), who were treated with neither PSL nor IFN- $\alpha$ at any point during the period of evaluation. Attributes of each group, such as sex, period of evaluation, age, and disease duration, were first evaluated to determine if any confounding variables were present.

Approximately $70 \%$ of all patients were female, and $30 \%$ were male. There was no significant difference between the sex ratio of the Steroids group and that of the Untreated group (Table 2). The overwhelming majority of patients in the Steroids group were monitored at St. Marianna University, whereas roughly equal numbers of untreated patients were monitored at St. Marianna University and at other institutions. Being monitored at St. Marianna University versus another institution had no significant (Wilcoxon/Kruskal-Wallis 2-sample test) effect on outcome within treatment groups.

Slightly more than half of all patients were evaluated for $>3$ years (Long-Term), and slightly less than half were evaluated for less than 3 years (Short-Term). The median period of evaluation for all patients was 3.4 years [interquartile range (IQR) 1.8-5.4 years]. There was no significant difference between the Steroids group and Untreated group regarding the percentage of patients evaluated in the Long-Term or Short-Term (Table 3). The median period of evaluation was 3.9 years (IQR 1.9-5 years) in the Steroids group and 3.0 years (IQR 1.6-6.1) in the Untreated group ( $\mathrm{p}=$ nonsignificant; Table 3 ).

The median ages and disease durations of the patients in each group at the onset of HAM/TSP, diagnosis of HAM/ TSP, beginning of the period of evaluation, and end of evaluation are shown in Fig. 1 ( $\mathrm{B}$ and $\mathrm{C}$, respectively). While patients in the Steroids group were slightly younger than those in the Untreated group, the differences were never significant. The patients in the Steroids group suffered from HAM/TSP for durations similar to or slightly longer than those in the Untreated group, and again these differences were not significant. At the beginning of the period of evaluation, patients were 58 years of age (IQR 52-65 years) in the Steroids group and 62 years of age (52-68 years) in the Untreated group ( $p=$ nonsignificant; Fig. 1B), and they had suffered from HAM/TSP for 12 years (6-17 years) in the Steroids group and 11 years (6-17.8 years) in the Untreated group $(p=$ nonsignificant; Fig. 1C). 
a

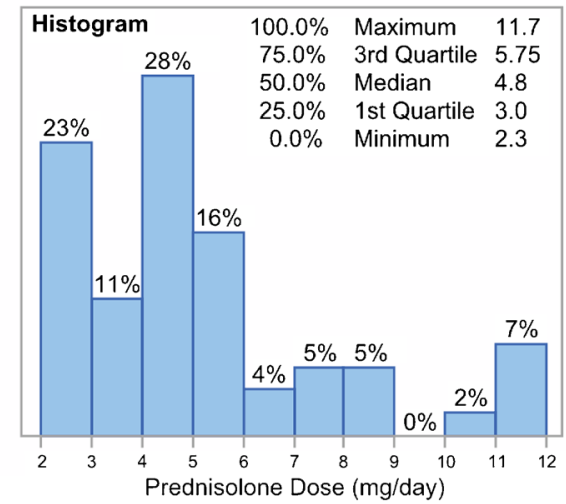

b
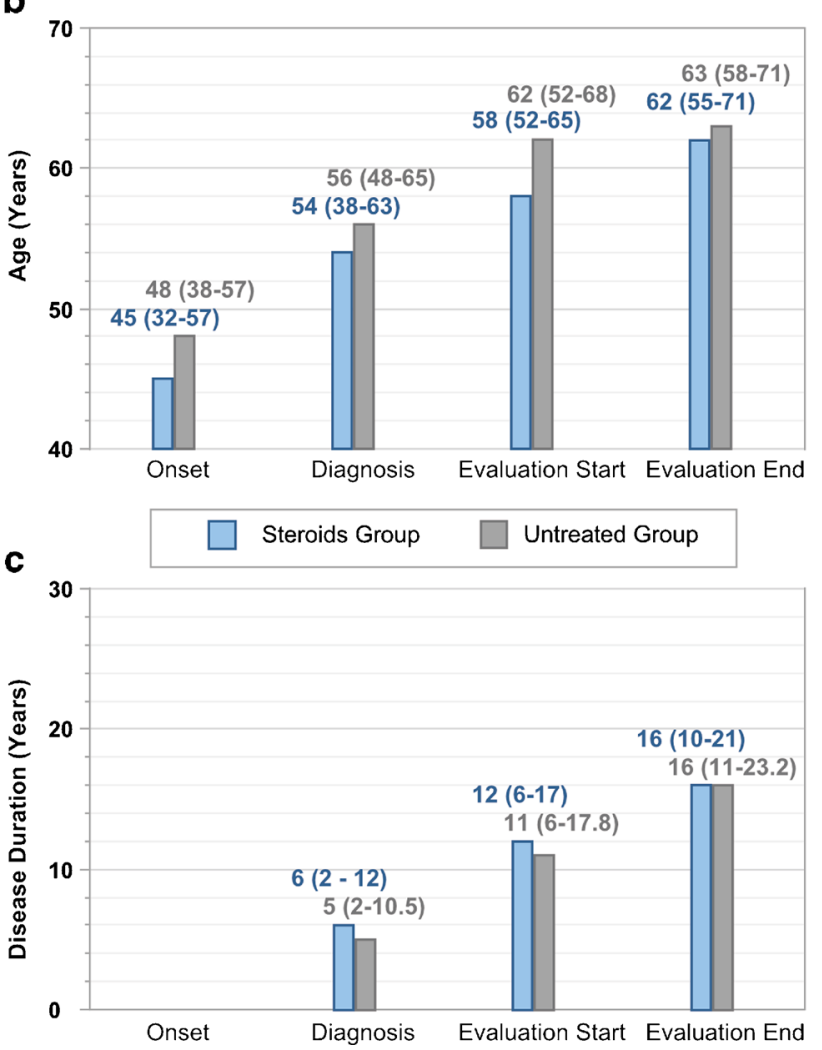

Fig. 1 Prednisolone (PSL) dose, age, and disease duration of study participants. (A) Distribution of the average daily dose of PSL in all treated patients $(n=57)$. (B, C) Median (and interquartile range) (B) age and $(\mathrm{C})$ disease duration of patients in the "Steroids" group (blue, $n=57$ ) and "Untreated" group (gray, $n=29$ ) at the onset of human Tlymphotropic virus type 1 (HAM)/tropical spastic paraparesis (TSP), diagnosis of HAM/TSP, and the beginning and end of the period of evaluation (listed from left to right in chronological order). There were no significant differences in age or disease duration between the patients in the Steroids and Untreated groups (Wilcoxon/Kruskal-Wallis 2-sample test, normal approximation)

\section{Changes in OMDS Experienced by Patients in the Steroids and Untreated Groups}

Treated patients were more likely to improve than their untreated counterparts $(p<0.01$; Fig. 2). Only 1 out of 29 untreated patients (3\%) improved, dropping from OMDS 4 to
OMDS 3 over 3 years (Fig. 2B). As many as 13 (45\%) worsened, and the majority (52\%) experienced no notable change in OMDS (Fig. 2D). By contrast, 20/57 (35\%) treated patients exhibited a decrease of at least 1 OMDS grade, including 5 (9\%) whose OMDS decreased by $\geq 2$ grades (Fig. 2A, C). However, 14/57 (25\%) worsened despite receiving constant treatment, and 23/57 (40\%) experienced no notable change in OMDS. The median score for treated patients remained unchanged at OMDS 5 over the course of the period of evaluation; the median score for untreated patients rose from OMDS 4 to OMDS 5 (Fig. 2A, B). To summarize, 57 patients were treated with low-dose PSL daily or on alternating days (about $5 \mathrm{mg} /$ day), and over one-third improved by at least 1 disability grade over a mean of 4 years.

\section{Short-Term and Long-Term Effects}

A sub-analysis was conducted to compare the effects of the treatment over Short-Term (defined as $<3$ years, $n=38$ ) and Long-Term $(>3$ years, $n=48)$ periods of evaluation. The median period was 1.7 years (IQR 1.1-2.1 years) for Short-Term and 5.1 years (IQR 4.3-6.8 years) for Long-Term evaluations (Table 3). As shown in Table 3, there were no significant differences between the Steroids and Untreated groups in terms of age or disease duration, even within the Short-Term and LongTerm subcategories. Similarly, Short-Term patients who were treated and untreated were evaluated for similar periods of time ( $\mathrm{p}=$ nonsignificant). However, among Long-Term patients, untreated patients were evaluated significantly longer than treated patients (6.1 years and 4.8 years, respectively; $p<0.05$ ).

Over both the Short-Term and Long-Term, treated patients were more likely to improve than their untreated counterparts ( $p<0.001$ for Short-Term; $p<0.05$ for Long-Term). As shown in Fig. 3(A-D), patients evaluated Short-Term were unlikely to worsen notably, regardless of treatment status: only $3 / 23(13 \%)$ treated patients and 2/15 (13\%) untreated patients exhibited an increase in OMDS. Over the Short-Term, as many as $12(52 \%)$ treated patients showed improvement (Fig. 3C), while none in the Untreated group did (Fig. 3D). The median motor disability declined from OMDS 6 to OMDS 5 in the Steroids group (Fig. 3A), whereas it started and remained at 5 in the Untreated group (Fig. 3B).

Over the Long-Term, 11/34 (32\%) treated patients and 11/ 14 (79\%) untreated patients suffered from a worsening of motor disability as indicated by an increase of $\geq 1$ OMDS grades (Fig. 3E-H). Many treated patients (44\%) experienced no notable change in motor ability (Fig. 3G), whereas only 2 (14\%) untreated patients were unchanged (Fig. 3H). While only 1 untreated patient improved, the motor symptoms of 8 (24\%) treated patients were alleviated enough to reduce the OMDS by at least 1 grade. The median OMDS remained constant at OMDS 5 for treated patients and rose from OMDS 3.5 to OMDS 4 for untreated patients (Fig. 3E, F). 
Table 2 Sex, monitoring institution, and period of evaluation for patients in the Steroids and Untreated groups

\begin{tabular}{|c|c|c|c|c|c|c|c|}
\hline & & \multicolumn{2}{|c|}{ Steroids } & \multicolumn{2}{|c|}{ Untreated } & \multicolumn{2}{|c|}{ Total } \\
\hline & & $n$ & $\%$ & $n$ & $\%$ & $n$ & $\%$ \\
\hline \multirow[t]{2}{*}{ Sex } & Female & 42 & 73.68 & 19 & 65.52 & 61 & 70.93 \\
\hline & Male & 15 & 26.32 & 10 & 34.48 & 25 & 29.07 \\
\hline \multirow[t]{2}{*}{ Institution } & St. Marianna & 50 & 87.72 & 14 & 48.28 & 64 & $74.42 *$ \\
\hline & Other & 7 & 12.28 & 15 & 51.72 & 22 & 25.58 \\
\hline \multirow[t]{2}{*}{ Period } & Short-Term & 23 & 40.35 & 15 & 51.72 & 38 & 44.19 \\
\hline & Long-Term & 34 & 59.65 & 14 & 48.28 & 48 & 55.81 \\
\hline Total & & 57 & 66.28 & 29 & 33.72 & 86 & 100.00 \\
\hline
\end{tabular}

Patients in the "Steroids" group were treated with prednisolone daily (or on alternating days), whereas those in the "Untreated" group were never treated during the period of evaluation. St. Marianna refers to St. Marianna University School of Medicine Hospital; period refers to the period of evaluation; Long-Term is defined as > 3 years; and Short-Term is defined as $<3$ years. Data are summarized using patient counts and percentages. $p$-values were calculated using Fisher's exact test (2-tailed)

*Significant $(p<0.001)$

\section{Effect of Baseline OMDS}

The mean baseline OMDS was 5 (IQR 4-7) in the Steroids group and $4(3-5)$ in the Untreated group $(p<0.01$, Wilcoxon/ Kruskal-Wallis 2-sample test). In order to assess the effect of baseline OMDS on outcomes, patients were stratified into 4 OMDS groups, and outcomes were compared within each stratum (Table 4). Patients in the Steroids group experienced significantly better outcomes than their counterparts in the
Untreated group even when compared within their baseline OMDS strata ( $p=0.019$, van Elteren test). This result held true both when compared between only Short-Term patients $(p=0.0091)$ or Long-Term patients $(p=0.031)$. Importantly, beneficial effects of PSL were not limited to patients with mild disease: $60 \%$ of patients with a baseline OMDS $>7$ improved with PSL treatment.

\section{Rate of OMDS Change}

The rate of OMDS change ( $\triangle \mathrm{OMDS} /$ year) was also calculated for each patient, as described previously by Osame et al. [4], and there was a mean change of -0.13 OMDS/year in the Steroids group and +0.12 OMDS/year in the Untreated group $(p<0.01$; Fig. 4). The distribution of rates was more positive in the Untreated group, even when evaluating only either Short-Term patients $(p<0.05)$ or Long-Term patients alone $(p<0.05$, Wilcoxon/Kruskal-Wallis 2-sample test, normal approximation).

\section{Discussion}

Our study takes a novel approach in several respects. First, we recorded the effects of PSL administered at a maintenance dose, a regimen designed to keep a small quantity of the drug in the body continuously. Our average daily dose of $4.8 \mathrm{mg} /$ day is roughly equivalent to the maintenance dose Osame et al. [4] transiently employed to taper patients from their initial dose (which was $>10$ times greater) ultimately down
Table 3 Age, disease duration, and period of evaluation for patient groups

\begin{tabular}{|c|c|c|c|c|c|c|c|}
\hline & \multicolumn{2}{|l|}{ Steroids } & \multicolumn{2}{|c|}{ Untreated } & \multicolumn{2}{|l|}{ All } & \\
\hline & Median & IQR & Median & IQR & Median & IQR & \\
\hline Age at start (y) & 58 & $52-66$ & 62 & $51-69$ & 59 & $52-67$ & \\
\hline Short Term $(n=38)$ & 58 & $53-64$ & 62 & $52-70$ & 59 & $53-66$ & \\
\hline Long Term $(n=48)$ & 58 & $52-67$ & 59 & $49-69$ & 58 & $51-67$ & \\
\hline Disease duration at start (y) & 12 & $6-17.5$ & 11 & $6-17.9$ & 11.8 & $6-17.9$ & \\
\hline Short Term $(n=38)$ & 9 & $6-19$ & 14 & $6-24$ & 12 & $6-19.3$ & \\
\hline Long Term $(n=48)$ & 12 & $6.3-15$ & 10 & $5.8-15.5$ & 11.3 & $6.3-15$ & \\
\hline Period of evaluation (y) & 3.9 & $1.9-5$ & 3 & $1.6-6.1$ & 3.4 & $1.8-5.4$ & \\
\hline Short Term $(n=38)$ & 1.6 & $1.1-2.1$ & 1.8 & $0.8-2.2$ & 1.7 & $1.1-2.1$ & \\
\hline Long Term $(n=48)$ & 4.8 & $4.1-6.1$ & 6.1 & $5.6-7.1$ & 5.1 & $4.3-6.8$ & * \\
\hline
\end{tabular}

Patients in the "Steroids" group were treated with prednisolone daily (or on alternating days), whereas those in the "Untreated" group were never treated during the period of evaluation. Period of evaluation refers to the time from start, i.e., the first visit (Untreated group) or first day of treatment (Steroids group), to end, i.e., the most recent visit before their data were transmitted, and this period is classified as either "Long Term" ( 3 years) or "Short term" $(<3$ years $)$

Data are summarized using the median and interquartile range. $p$-values were calculated using the Wilcoxon/ Kruskal-Wallis test (2-sample test, normal approximation)

* Significant at $p<0.05$

Disease duration $=$ period from disease onset to start; IQR $=$ interquartile range 


\section{a STEROIDS}

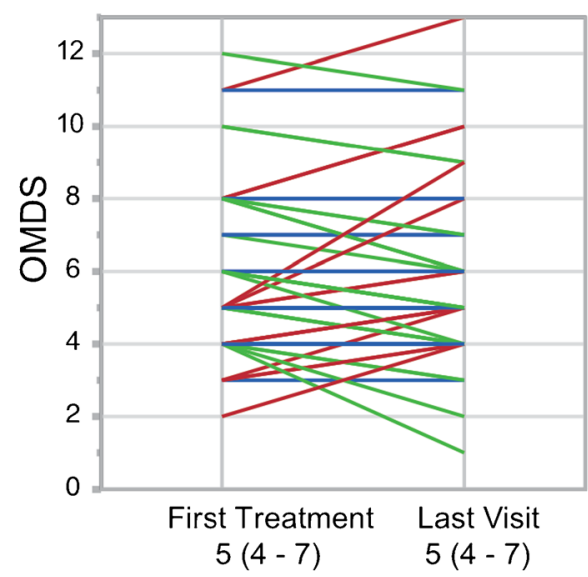

C

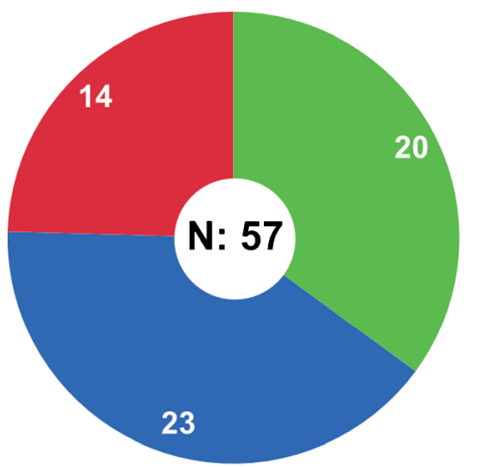

OMDS CHANGE: IMPROVED

Fig. 2 Change in motor disability in the "Steroids" vs "Untreated" groups over the period of evaluation. (A, B) Parallel plots and (C, D) pie graphs illustrating the changes in Osame Motor Disability Score (OMDS) experienced by patients in the (A, C) steroids $(n=57)$ and $(\mathrm{B}$, D) untreated $(n=29)$ groups from the beginning to the end of the period of evaluation. The same color code applies to both the parallel plots and pie graphs: green for improved, blue for unchanged, and red for worsened OMDS. In the parallel plots, each patient is represented by a line drawn between the OMDS at the start of evaluation (on the date of the first treatment for patients given steroids; on the date of the first visit for untreated patients) and the OMDS at the end of evaluation (on the latest

to 0 . The implication of the study by Osame et al. [4] and other similar studies is that the initial dose exerts a positive therapeutic effect and the maintenance dose merely helps extend that effect. Here we observed the effects of the maintenance dose itself over time. Owing to such differences in dose, as well as in duration of the study, it is not meaningful to directly compare our results with those of previous investigations.

A second novel aspect of our study is that we evaluated the effects of PSL on a group of patients by comparing their progress against that of an Untreated group. Previous studies evaluated the effects of PSL on their patients by looking for substantial improvements $[4,5,16,17,19,20,25-28]$, whereas we also looked for an absence or slowing of disease progression associated with the treatment. Patients were prescribed treatment or b UNTREATED

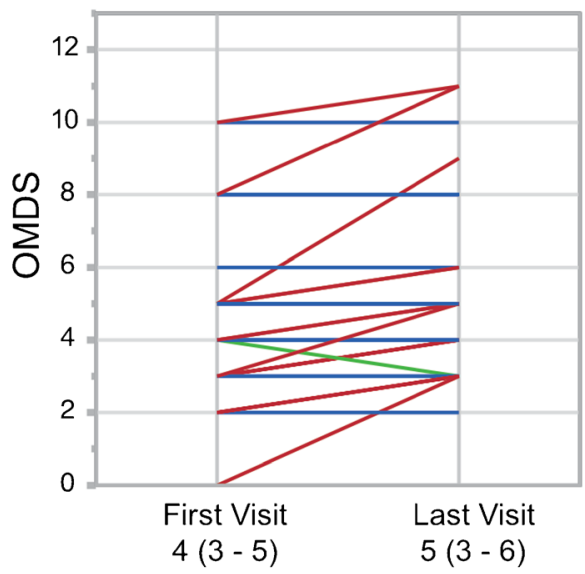

d

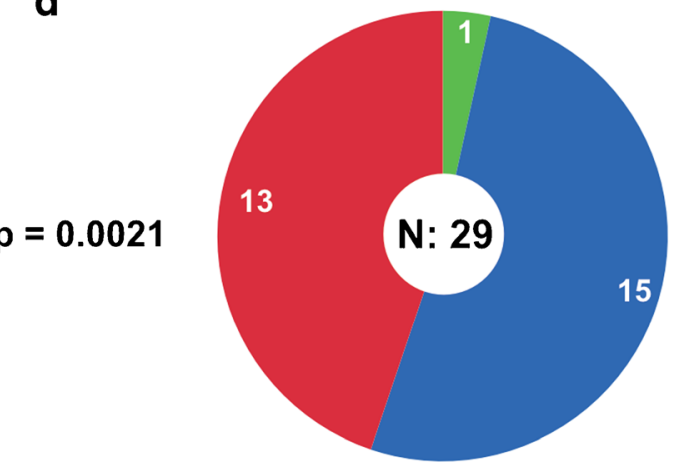

NO CHANGE WORSENED

visit with the attending physician before their data was transmitted). In the case that identical OMDS values were recorded for multiple patients, the lines representing those patients overlap and appear as a single line. The median OMDS (and interquartile range) for each group at each time point is noted below the parallel plots. While the parallel plots show the magnitude of change in OMDS, the pie graphs show the number of patients who experienced any improvement or worsening in OMDS. Patients in the Steroids group were significantly more likely to improve than those in the Untreated group $(p<0.01)$. The $p$-value was calculated using Fisher's exact test (2-tailed)

left untreated by their physicians based on their individual needs and preferences. In other words, we did not sort patients into the two groups controlling for confounding variables such as age, sex, or disease duration. Instead, we retrospectively analyzed the two groups to assess the presence or absence of significant differences in those variables, and we found none.

We did note, however, that the mean OMDS at the start of evaluation was lower in the Untreated group (OMDS $4 \mathrm{vs}$ OMDS $5 ; p<0.01)$, which was to be expected: physicians are more likely to risk the side effects of daily steroids to treat patients exhibiting more severe HAM/TSP symptoms. However, when we performed a stratified analysis controlling for baseline OMDS, we found that the steroids retained their significant beneficial effects. 
Fig. 3 Change in motor disability in the "Steroids" vs "Untreated" groups over short- and LongTerm periods. (A, B, E, F) Parallel plots and $(\mathrm{C}, \mathrm{D}, \mathrm{G}, \mathrm{H})$ pie graphs illustrating the changes in Osame Motor Disability Score (OMDS) experienced by patients in the Steroids $(n=57$, left) and Untreated ( $n=29$, right) groups from the beginning to the end of the period of evaluation, here shown grouped by the length of that period (Short-Term above, Long-Term below). The same color code applies to both the parallel plots and pie graphs: green for improved, blue for unchanged, and red for worsened OMDS. In the parallel plots, each patient is represented by a line drawn between the OMDS at the start of evaluation (on the date of the first treatment for patients given steroids; on the date of the first visit for untreated patients) and the OMDS at the end of evaluation (on the latest visit with the attending physician before their data was transmitted). In the case that identical OMDS values were recorded for multiple patients, the lines representing those patients overlap and appear as a single line. The median OMDS (and interquartile range) for each group at each time point is noted below the parallel plots. While the parallel plots show the magnitude of change in OMDS, the pie graphs show the number of patients who experienced any improvement or worsening in OMDS. Patients in the Steroids group were significantly more likely to improve than those in the Untreated group, both over (C, D) the Short-Term $(p<0.001)$ and $(\mathrm{G}, \mathrm{H})$ the Long-Term $(p<0.05)$. The $p$-values were calculated using Fisher's exact test (2-tailed)
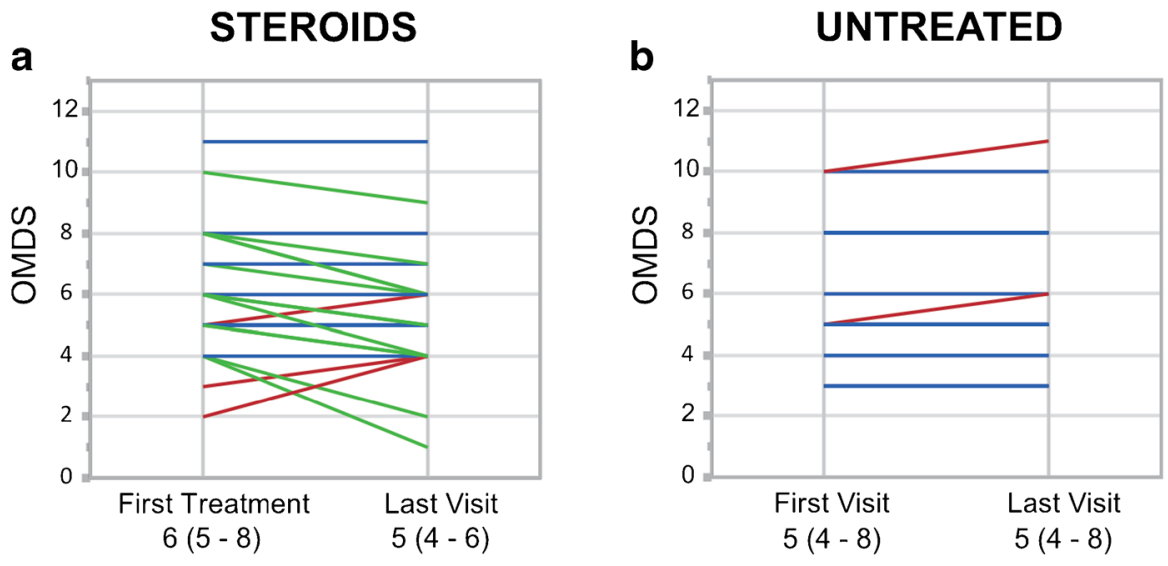

C
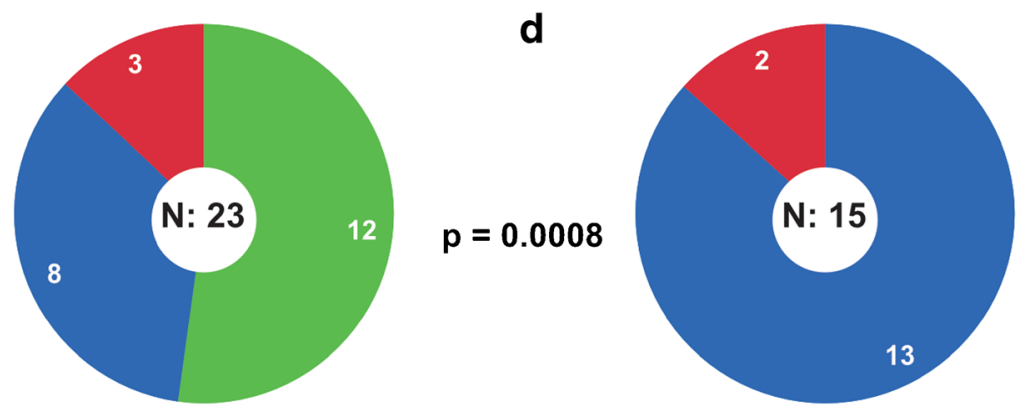

\section{e}

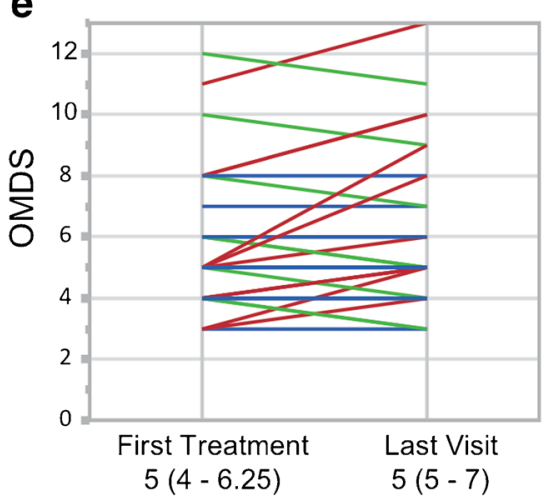

f

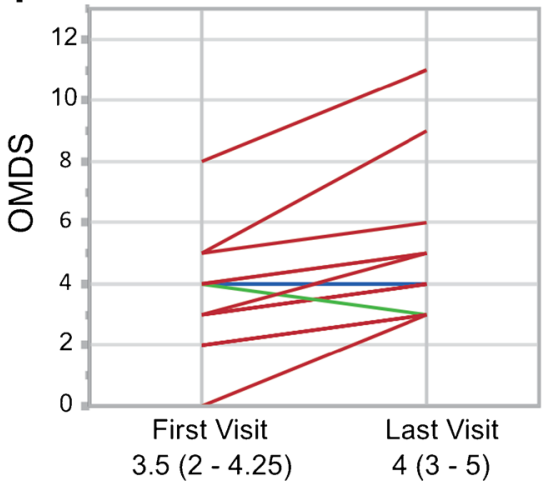

h
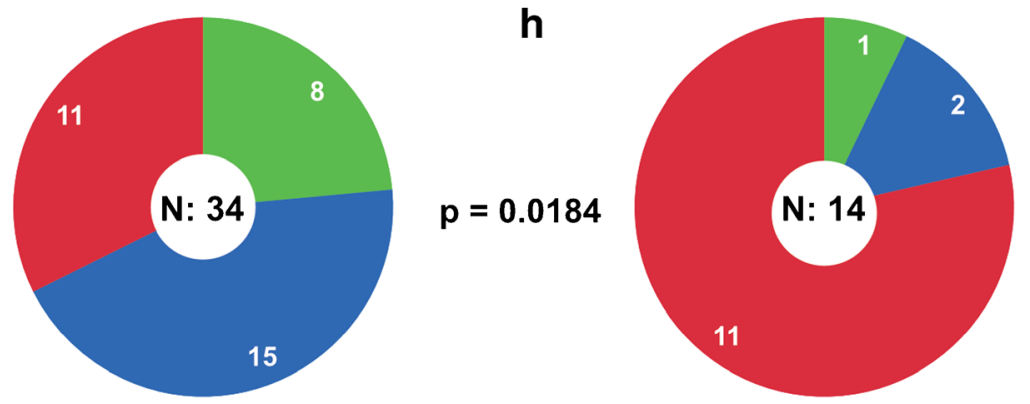

OMDS CHANGE: IMPROVED

NO CHANGE WORSENED

We also noticed that most treated patients were monitored at the St. Marianna University clinic, which likely reflects that patients experiencing severe symptoms chose to travel to this clinic, even when other hospitals were closer, to seek counsel from the HAM/TSP specialists there. Clinic choice had negligible effect on outcomes within 
treatment groups, and we do not consider it to be a confounding variable.

Owing to the study design, we were, of course, unable to administer a placebo drug to the Untreated group. This inability to account for the placebo effect is a natural limitation of many observational and retrospective studies, which are necessarily open-label. One method of overcoming this limitation is to compare the effects of PSL with those of other therapies, as Nakagawa et al. [20] have done. While we did receive some reports of patients treated with IFN- $\alpha$ or other therapies, there were not nearly enough patients treated continuously with a rival therapy to constitute a group for statistical comparisons. Even though our study falls short of the gold-standard, double-blind, placebo-controlled trial, our findings provide the most robust evidence to date of the effectiveness of oral corticosteroids for slowing the progression of HAM/TSP.

The third, and final, novel aspect of this study is the emphasis on the effects of Long-Term systemic corticosteroid use on patients with HAM/TSP, specifically longer than 3 years, which had never been evaluated before. The fact that most patients treated with PSL did not experience a worsening of motor disability over the long-term (roughly 5 years) was remarkable for a disease known to be chronically progressive. Indeed, our own data showed that patients left untreated for similar periods of time (roughly 6 years) almost always (11/14 patients) progressed by at least 1 OMDS grade. Unfortunately, the untreated patients in the Long-Term category happened to be evaluated slightly longer than their treated counterparts $(6.1$ vs $4.8 ; p<0.05$ ), and this constitutes a confounding variable. However, it is generally well established that patients with HAM/TSP gradually become worse over time, not better

Table 4 Outcomes by Baseline Osame Motor Disability Score (OMDS)

\begin{tabular}{|c|c|c|c|c|c|c|c|c|}
\hline & \multirow[t]{2}{*}{ Baseline OMDS } & \multicolumn{2}{|c|}{ Improved } & \multicolumn{2}{|c|}{ No Change } & \multicolumn{2}{|c|}{ Worsened } & \multirow{2}{*}{$\begin{array}{l}\text { Total } \\
n\end{array}$} \\
\hline & & $n$ & $(\%)$ & $n$ & $(\%)$ & $n$ & $(\%)$ & \\
\hline \multirow[t]{4}{*}{ Steroids } & 3 or 4 & 4 & 27 & 5 & 33 & 6 & 40 & 15 \\
\hline & 5 & 3 & 18 & 10 & 59 & 4 & 24 & 17 \\
\hline & 6 or 7 & 6 & 55 & 5 & 45 & 0 & 0 & 11 \\
\hline & $\geq 8$ & 6 & 60 & 2 & 20 & 2 & 20 & 10 \\
\hline \multirow[t]{4}{*}{ Untreated } & 3 or 4 & 1 & 8 & 6 & 50 & 5 & 42 & 12 \\
\hline & 5 & 0 & 0 & 4 & 57 & 3 & 43 & 7 \\
\hline & 6 or 7 & 0 & 0 & 1 & 100 & 0 & 0 & 1 \\
\hline & $\geq 8$ & 0 & 0 & 3 & 60 & 2 & 40 & 5 \\
\hline
\end{tabular}

Patients in the "Steroids" group were treated with prednisolone daily (or on alternating days), whereas those in the "Untreated" group were never treated during the period of evaluation. Data are summarized using patient counts and row percentages. Baseline OMDS refers to the OMDS at the start of each patient's evaluation. Outcomes are classified as improved (decrease in OMDS), no change, or worsened (increase in OMDS). The Steroids and Untreated groups were compared using the van Elteren test with significance set at $p<0.05$, and the resulting $p$-value was 0.019 (see reviews of HAM/TSP $[3,15]$ ), and it is therefore meaningful that one-quarter of these treated patients not only managed to avoid deteriorating, but actually improved by at least 1 OMDS grade. In the present study, with the exception of only one extreme outlier (Fig. 4), no untreated patients improved in either the Long-Term or the Short-Term group.

It should be noted that not only was this study national in scope, but the data suggest that our patients were a nationally representative sample: demographics and clinical features were similar between this study and our nationwide epidemiological study, in which $74.2 \%$ of respondents were female and reported an age of onset of 45 years old, age at diagnosis of 53 years, age at presentation of 63 years, and disease duration of 17 years [30]. Thus, though not without its limitations, our study suggests that PSL effectively slows, stops, or even somewhat reverses the course of HAM/TSP when taken continuously over several years, and we believe our results are applicable to the general HAM/TSP patient population.

In conclusion, our study addressed for the first time the effects of daily (or alternatingly daily) oral corticosteroids in patients with HAM/TSP 1) at a low or "maintenance" dose; 2) compared with an Untreated group; and 3) over both the longand short-term. We aimed to help settle the controversy over this treatment's efficacy, and we have made great progress, though we still have not firmly established the treatment using

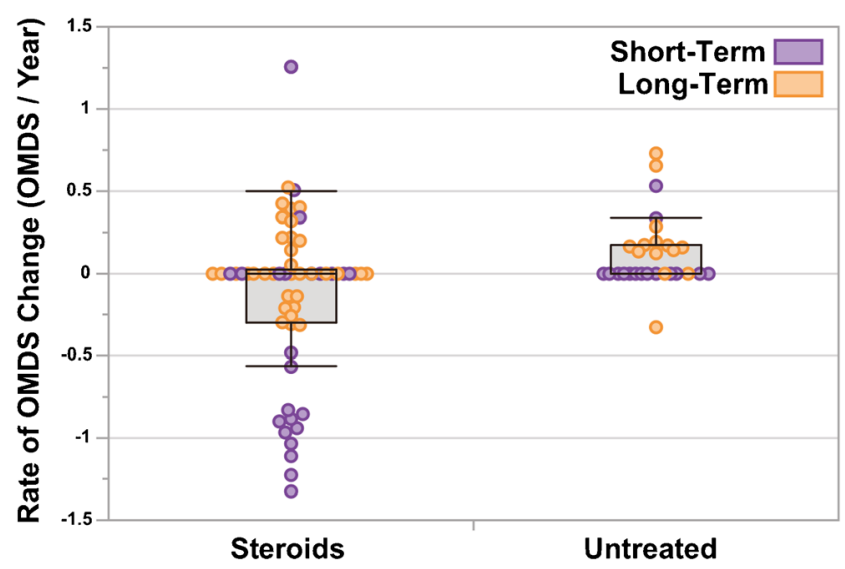

Fig. 4 Rate of change in motor disability for patients in the "Steroids" vs "Untreated" groups. Box-and-whisker plots showing the rate of change in Osame Motor Disability Score (OMDS) over time ( $\triangle$ OMDS/year) for patients in the Steroids $(n=57)$ and Untreated $(n=29)$ groups. Each point represents a single patient. Points are colored purple for patients evaluated over the Long-term ( $>3$ years) and orange for the Short-term $(<3$ years). The rate of change in OMDS was calculated for each patient using the quotient: total change in OMDS over the period of evaluation/the duration of the period of evaluation in years. The distributions for the Steroid and Untreated groups shown were significantly different $(p<0.01)$, as determined using the Wilcoxon/Kruskal-Wallis test (normal approximation). Points are jittered for ease of viewing. In the box-and-whisker plots, the boxes extend from the lower quartile to upper quartile and are segmented by a line representing the median, which in this case is at 0 for both groups. The whiskers extend to the maximum and minimum values, excluding outliers (more than $3 / 2$ times the value of the upper quartile or less than $3 / 2$ the value of the lower quartile) 
a placebo-controlled, double-blind clinical trial. Future studies must also be performed to clarify the relative effectiveness of PSL compared with other therapies, including IV mPSL, IFNs, steroid-sparing immunosuppressive drugs, antiretrovirals, monoclonal antibodies, and others (reviewed elsewhere [3, $31,32]$ ). For our part, we found PSL to be considerably effective compared with nontreatment, and we advocate for its use to slow disease progression. That said, the preponderance of positive outcomes in the short-term over long-term reaffirms the principle that this treatment is designed to delay, not cure, the disease. Since the first of these PSL studies was performed on patients with HAM/TSP in the 1980s, researchers have elucidated much of the once-mysterious pathogenesis of HAM/ TSP, beginning with advent of the theory of bystander damage in 1993 [33]. While the catastrophic deregulation of the immune system is, indeed, central to the pathomechanism, it has also been suggested that specifically targeting the virusinfected T cells may now be possible [34], may be more effective, and may even constitute a cure, and a clinical trial testing such a drug (mogamulizumab [35]) is now underway at St. Marianna University. In the meantime, we are pleased to recommend long-term, low-dose daily PSL to reduce serious bystander damage in patients who tolerate the side effects well.

Acknowledgements This work was supported by the Japan Agency for Medical Research and Development (AMED; www.amed.go.jp), the Ministry of Health Labour and Welfare (www.mhlw.go.jp), and by the Japan Society for the Promotion of Science KAKENHI (grant no. 16H05323; https://www.jsps.go.jp/index.html). The funders had no role in study design, data collection and analysis, decision to publish, or preparation of the manuscript.

Required Author Forms Disclosure forms provided by the authors are available with the online version of this article.

\section{References}

1. Gessain A, Cassar O. Epidemiological aspects and world distribution of HTLV-1 infection. Front Microbiol 2012;3:388.

2. Satake M, Yamaguchi K, Tadokoro K. Current prevalence of HTLV-1 in Japan as determined by screening of blood donors. J Med Virol 2012;84:327-335.

3. Bangham CRM, Araujo A, Yamano Y, et al. HTLV-1-associated myelopathy/tropical spastic paraparesis. Nat Rev Dis Prim 2015;1: 15012.

4. Osame M, Igata A, Matsumoto M, et al. HTLV-I-associated myelopathy (HAM), treatment trials, retrospective survey and clinical laboratory findings. Hematol Rev 1990;3:271-84.

5. Osame M, Usuku K, Izumo S, et al. HTLV-I associated myelopathy, a new clinical entity. Lancet 1986;1:1031-1032.

6. Gessain A, Vernant JC, Maurs L, et al. Antibodies to human Tlymphotropic virus type-I in patients with tropical spastic paraparesis. Lancet 1985;326:407-410.

7. Román GC, Osame M. Identity of HTLV-I-associated tropical spastic paraparesis and HTLV-I-associated myelopathy. Lancet 1988;1:651.

8. Mani KS, Mani AJ, Montgomery RD. A spastic paraplegic syndrome in South India. J Neurol Sci 1969;9:179-199.
9. Montgomery RD, Cruickshank EK, Robertson WB, et al. Clinical and pathological observations on Jamaican neuropathy; a report on 206 cases. Brain 1964;87:425-462.

10. Barnes PJ. How corticosteroids control inflammation: Quintiles Prize Lecture 2005. Br J Pharmacol 2006;148:245-254.

11. Liu D, Ahmet A, Ward L, et al. A practical guide to the monitoring and management of the complications of systemic corticosteroid therapy. Allergy, Asthma, Clin Immunol 2013;9:30.

12. Compston A. Methylprednisolone and multiple sclerosis. Arch Neurol 1988;45:669-670.

13. Mertin J, Kremer M, Knight SC, et al. Double-blind controlled trial of immunosuppression in the treatment of multiple sclerosis: final report. Lancet 1982;320:351-354.

14. Newman PK, Saunders M, Tilley PJ. Methylprednisolone therapy in multiple sclerosis. J Neurol Neurosurg Psychiatry 1982;45:941-942.

15. Yamano Y, Sato T. Clinical pathophysiology of human Tlymphotropic virus-type 1-associated myelopathy/tropical spastic paraparesis. Front Microbiol 2012;3:1-10.

16. Osame M, Matsumoto M, Usuku K, et al. Chronic progressive myelopathy associated with elevated antibodies to human Tlymphotropic virus type I and adult T-cell leukemialike cells. Ann Neurol 1987;21:117-122.

17. Vernant JC, Maurs L, Gessain A, et al. Endemic tropical spastic paraparesis associated with human T-lymphotropic virus type I: a clinical and seroepidemiological study of 25 cases. Ann Neurol 1987;21:123-130.

18. Höllsberg P, Hafler DA. Seminars in medicine of the Beth Israel Hospital, Boston. Pathogenesis of diseases induced by human lymphotropic virus type I infection. N Engl J Med 1993;328: 1173-1182.

19. Araújo AQ, Afonso CR, Leite AC, et al. Intravenous methylprednisolone in HTLV-I associated myelopathy/tropical spastic paraparesis (HAM/TSP). Arq Neuropsiquiatr 1993;51:325-328.

20. Nakagawa M, Nakahara K, Maruyama Y, et al. Therapeutic trials in 200 patients with HTLV-I-associated myelopathy/tropical spastic paraparesis. J Neurovirol 1996;2:345-355.

21. Bhagavati S, Ehrlich G, Kula RW, et al. Detection of human T-cell lymphoma/leukemia virus type I DNA and antigen in spinal fluid and blood of patients with chronic progressive myelopathy. N Engl J Med 1988;318:1141-1147.

22. Rodgers-Johnson P, Morgan OSC, Mora C, et al. The role of HTLVI in tropical spastic paraparesis in Jamaica. Ann Neurol 1988;23: S121-S126.

23. Gout $\mathrm{O}$, Gessain A, Bolgert F, et al. Chronic myelopathies associated with human T-lymphotropic virus type I. A clinical, serologic, and immunovirologic study of ten patients in France. Arch Neurol 1989;46:255-260.

24. Zaninovic V, Arango C, Biojo R, et al. Tropical spastic paraparesis in colombia. Ann Neurol 1988;23:S127-S132.

25. Cruickshank JK, Rudge P, Dalgleish AG, et al. Tropical spastic paraparesis and human $\mathrm{T}$ cell lymphotropic virus type 1 in the United Kingdom. Brain 1989;112:1057-1090.

26. Duncan J, Rudge P. Methylprednisolone therapy in tropical spastic paraparesis. J Neurol Neurosurg Psychiatry 1990;53:173-174.

27. Kira J, Fujihara K, Itoyama Y, et al. Leukoencephalopathy in HTLV-I-associated myelopathy/tropical spastic paraparesis: MRI analysis and a two year follow-up study after corticosteroid therapy. J Neurol Sci 1991;106:41-49.

28. Croda MG, de Oliveira ACP, Vergara MPP, et al. Corticosteroid therapy in TSP/HAM patients: the results from a 10 years open cohort. J Neurol Sci 2008;269:133-137.

29. De Castro-Costa CM, Araújo AQC, Barreto MM, et al. Proposal for diagnostic criteria of tropical spastic paraparesis/HTLV-I-associated myelopathy (TSP/HAM). AIDS Res Hum Retroviruses 2006;22: 931-935. 
30. Coler-Reilly ALG, Yagishita N, Suzuki H, et al. Nation-wide epidemiological study of Japanese patients with rare viral myelopathy using novel registration system (HAM-net). Orphanet J Rare Dis 2016;11.

31. Martin F, Taylor GP, Jacobson S. Inflammatory manifestations of HTLV-1 and their therapeutic options. Expert Rev Clin Immunol 2014;10:1531-1546.

32. Araújo A, Lima MA, Silva MTT. Human T-lymphotropic virus 1 neurologic disease. Curr Treat Options Neurol 2008;10:193-200.

33. Ijichi S, Izumo S, Eiraku N, et al. An autoaggressive process against bystander tissues in HTLV-1-infected individuals - a possible pathomechanism of HAM/TSP. Med Hypotheses 1993;41:542547.

34. Yamano Y, Coler-Reilly A. HTLV-1 induces a Th1-like state in CD4 + CCR4+ T cells that produces an inflammatory positive feedback loop via astrocytes in HAM/TSP. J Neuroimmunol 2017;304: 51-55.

35. Yamauchi J, Coler-Reilly A, Sato T, et al. Mogamulizumab, an antiCCR4 antibody, targets human T-lymphotropic virus type 1infected CD8+ and CD4+ T cells to treat associated myelopathy. J Infect Dis 2015;211:238-248. 